\title{
Combinatorial investigation of the isolated nanoparticle to coalescent layer transition in a gradient sputtered gold nanoparticle layer on top of polystyrene
}

\author{
S. V. Roth ${ }^{\text {a) }}$ \\ HASYLAB at DESY, Notkestr. 85, D-22603 Hamburg, Germany \\ H. Walter \\ CSEM SA, Badenerstrasse 569, CH-8048 Zürich, Switzerland
}

M. Burghammer and C. Riekel

ESRF, 6 rue Jules Horowitz, F-38043 Grenoble, France

B. Lengeler, C. Schroer, ${ }^{\text {b) }}$ and M. Kuhlmann ${ }^{\text {b) }}$

2. Phys. Inst. B, D-52056 Aachen, Germany

T. Walther and A. Sehrbrock

Forschungszentrum caesar, Ludwig-Erhard-Allee 2, D-53175 Bonn, Germany

R. Domnick

identif GmbH, Ulrich-Schalk-Str. 3, D-91056 Erlangen, Germany

\author{
P. Müller-Buschbaum \\ Physik-Department E13, TU München, James-Franck-Str. 1, D-85748 Garching, Germany
}

(Received 29 September 2005; accepted 7 December 2005; published online 12 January 2006)

\begin{abstract}
Within a combinatorial investigation, a gradient sputtered gold layer on top of polystyrene on silicon substrate is addressed. Results from a real-space inspection by transmission electron microscopy are compared with surface-sensitive microbeam grazing incidence small-angle x-ray scattering. The combinatorial approach allows distinguishing different morphologies prepared under exactly the same environmental conditions on one single substrate. The transition of a coalescent layer to an isolated nanoparticle layer is determined as a function of sputter rate. Though optical spectra show only slight differences, the morphology and structure are distinctly different from evaporated layers prepared with same mass thickness. (C) 2006 American Institute of Physics.
\end{abstract}

[DOI: 10.1063/1.2161926]

Metallic nanometer-sized particles show unexpected properties which are distinctly different from the properties of the bulk material. ${ }^{1}$ Due to their high potential in catalysis ${ }^{2}$ and organic solar cells ${ }^{3,4}$ such particles are the subject of basic research as well as technological applications. ${ }^{5}$ Some applications, including security features and biochips, already combine metallic nanoparticle layers with polymer layers in multilayer setups as summarized by Bauer et al.. ${ }^{6}$ For large-area applications, vacuum deposition of metallic nanoparticles is the most widely used technique due to its relative simplicity, and characteristic length scales below 10 $\mathrm{nm}$ can be obtained. ${ }^{7}$ Combinatorial studies are of utmost importance in materials science and engineering, e.g., for optimization of organic light-emitting devices. ${ }^{8}$ Samples adapted to combinatorial mapping include single- or multidimensional gradients. They allow for distinguishing different morphologies prepared under exactly the same environmental conditions on one single substrate. Gradient layers offer the possibility to study the influence of deposition rate while keeping all other parameters constant. ${ }^{9}$ Specifically, the question of coalescence and the transition to isolated nanoparticles showing pronounced plasmon resonances can be addressed as being important, e.g., in

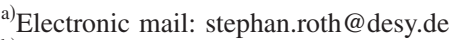

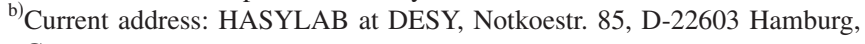
Germany.
}

anticounterfeiting. ${ }^{10,6}$ The formation of the nanoparticle layer depends on many parameters, e.g., deposition technique and substrate temperature. Thus, a layer prepared to have the same nominal parameters but by sputtering instead of evaporation can possess distinctly different optical and electrical properties.

In this study, we focus on the morphological and structural properties of a sputtered gradient gold (Au) nanoparticle/polystyrene (PS)/silicon (Si) substrate multilayer system. Though optical properties are very similar, it turns out that its morphological properties are distinctly different from evaporated gradient layers with same mass thickness investigated in Ref. 9. The sample was prepared by ac- sputtering. Under standard sputtering conditions, nominally $8 \mathrm{~nm}$ mass thickness of Au were deposited onto a PS film of $40 \mathrm{~nm}$ thickness on Si substrate at room temperature in a vacuum chamber (Sputter-Coater, tectra $\mathrm{GmbH}$ ). The same coater was used in Ref. 9 for preparation of evaporated samples. The sample was partly shadowed by a thin plate at about $0.5 \mathrm{~mm}$ distance from the PS layer, thereby introducing a lateral gradient in particle size. By diffusion of the gold atoms and self-assembly - the gold-gold interaction is much stronger than the gold-polymer interaction ${ }^{11}$ - a nanoparticle layer forms. Due to the much higher kinetic energy of sputtered $\mathrm{Au}$ atoms compared to evaporated atoms, the selfassembly process is different even at the same deposition rate. Figure 1 compares optical spectra in the visible range 


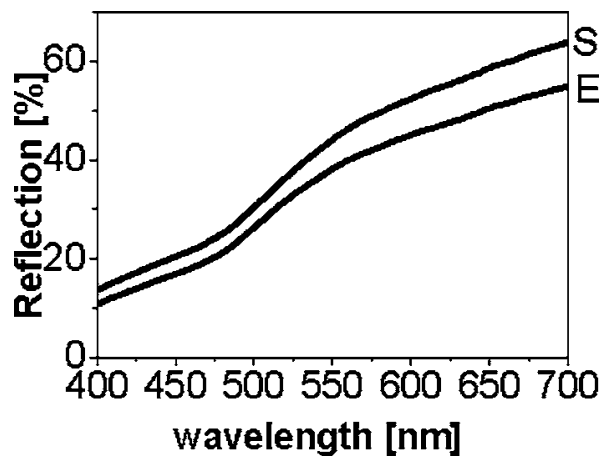

FIG. 1. Optical reflection spectra measured at $18^{\circ}$ and at an Au layer thickness of $8 \mathrm{~nm}$ as a function of wavelength in the visible regime. $\mathrm{S}$ denotes the sputtered and $\mathrm{E}$ is the evaporated $\mathrm{Au} / \mathrm{PS} / \mathrm{Si}$ multilayer.

from the sputtered sample (index: S)—obtained in reflection geometry using a Steak Microparts spectrometer-to that of the evaporated sample investigated in Ref. 9 (index: E). Clearly, the optical spectra of the two samples follow the same functional behavior in the visible regime. Such a behavior might lead to the conclusion that the nanostructure of the layers prepared by the two different deposition methods under standard conditions is comparable in morphology. To further address this important question, we used the advanced technique of microbeam grazing incidence smallangle $\mathrm{x}$-ray scattering ( $\mu \mathrm{GISAXS})$ as an invasive nondestructive scanning technique with high statistical relevance. $^{9,12}$ Scanning electron microscopy (SEM) and transmission electron microscopy (TEM) were operated to obtain a real-space image for comparison. Contrary to what might be deduced from Fig. 1, the two deposition methods yield different morphologies. The $\mu$ GISAXS experiment was performed at the beamline ID13/ESRF, with the same basic setup as in Ref. 9. The x-ray beam (size $5 \mu \mathrm{m}$ ) impinges on the sample under an incident angle of $\alpha_{i}=0.81^{\circ}$. The gradient was placed perpendicular to the beam and scanned at different positions y using a step width of $\Delta y=20 \mu \mathrm{m}$.

Figure 2(a) shows two representative two-dimensional (2D) $\mu$ GISAXS patterns of sample $\mathrm{S}$ obtained at $y_{1}$ $=620 \mu \mathrm{m}$ and $y_{2}=860 \mu \mathrm{m}$ together with the corresponding simulations. The beginning of the gradient is situated at $y$ $=0 \mu \mathrm{m}$ in the coalescent layer. The $2 \mathrm{D}$ intensity distribution is plotted as a function of $q_{y}=2 \pi / \lambda \sin (2 \theta)$ and $q_{z}$ $=2 \pi / \lambda\left[\sin \left(\alpha_{i}\right)+\left(\sin \left(\alpha_{f}\right)\right] .2 \theta\right.$ denotes the out-of-plane angle, $\alpha_{f}$ the exit angle, respectively. The 2D scattering pattern allows extracting the nanoparticle geometry parallel and vertical to the surface, i.e., radius $R$, distance $\xi$, height $H$ at each scan position $y .{ }^{13}$ In Fig. 2(b), we show a sketch of the gradient to visualize the geometry of the nanoparticle layer deduced from the $\mu$ GISAXS pattern. To analyze the $2 \mathrm{D}$ pattern, we used a modelling approach using the program IsGISAXs ${ }^{14}$ which considers two particle classes, namely cylindrical and ellipsoidal particles. Using simpler models did not give satisfactory results. Following Fig. 2(a), the position of the minima along $q_{z}$ (horizontal lines), as well as the position and form of the side maxima at $q_{y}$ (red arrows), can be excellently reproduced for all position along the whole gradient.

As the most relevant parameters for modeling, Fig. 2(c) shows the mean height $H$, the mean in-plane radius $R$ and mean in-plane distance $\xi$ as a function of the scan position $y$. From linear fits it is possible to group all parameters $H, R$, Downloaded 31 Aug 2007 to 131.169.95.147. Redistribution subject

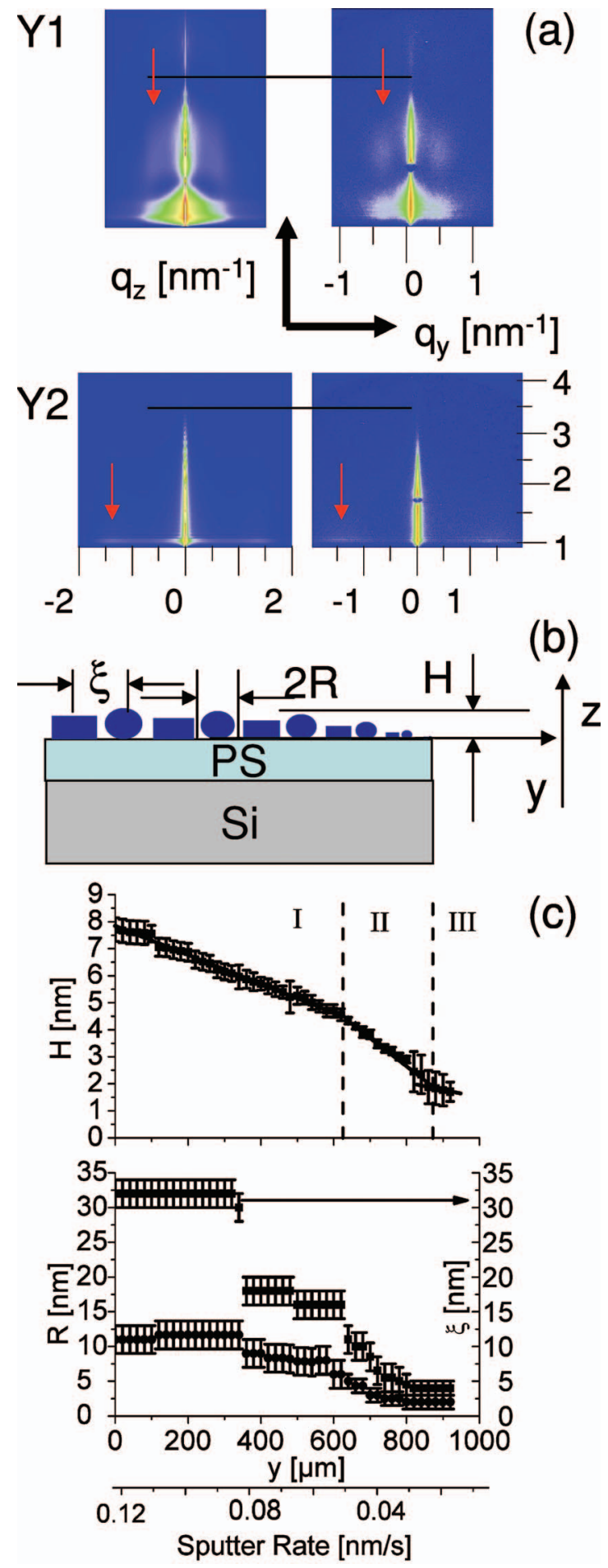

FIG. 2. (Color) (a) $\mu$ GISAXS data (right) and simulation (left) at selected scan positions $(y=620,860 \mu \mathrm{m}$, denoted Y1, Y2) measured for the sputtered sample S. Y1 is characteristic of a coalescent nanoparticle layer, while Y2 is characteristic of an isolated nanoparticle layer. Red arrows indicate the position of the side maxima, horizontal lines those of the minima. (b) Sketch (side view) of the gradient derived from the modeling. $\mathrm{H}$ denotes the nanoparticle height, $2 R$ their diameter, and $\xi$ their in-plane distance. (c) Characterization of the gradient in terms of $H, \xi$ (right ordinate, upper curve), and $R$ (left ordinate, lower curve) as a function of the scan position $y$. The linear fits in the curve $H$ vs $y$ show three distinct nanoparticle growth regimes (denoted I, II, III) with two cross-over points at $y=840 \mu \mathrm{m}$ and $y$ $=610 \mu \mathrm{m}$. The second ordinate correlates the scan position $y$ with the sputter rate.

and $\xi$ in three regions. These three regions can be correlated to different growth mechanisms showing a transition from a coalescent to isolated nanoparticle layer. To pursue our idea to AlP license or copyright, see http://apl.aip.org/apl/copyright.jsp 
(a)

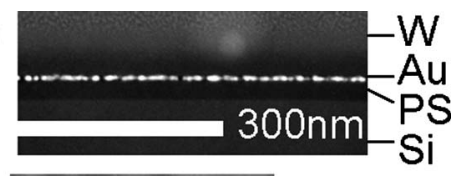

(b)

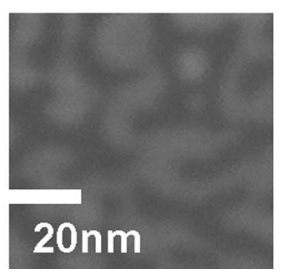

FIG. 3. (a) Cross-sectional transmission electron micrograph of a lamella cut by a FIB at $y=400 \mu \mathrm{m}$. Au denotes the nanoparticle layer, $\mathrm{W}$ a protective layer, PS the polystyrene layer and Si the silicon substrate. (b) Planview topograph of the surface at $y=400 \mu \mathrm{m}$ observed by secondary electrons in a SEM.

further, we correlate in Fig. 2(c) the sputter rate and local position $y$, thus enabling to probe different sputter rates at the early stages of nanoparticle layer formation while keeping all other parameters constant. In region I large nanoparticles (several $10 \mathrm{~nm}$ diameters) with distances comparable to their diameter prevail. This can be understood in terms of a Vollmer-Weber growth, subsequent Ostwald ripening, and coalescence of the nanoparticles, leading to rather large nanoparticles. Figure 3(a) shows the TEM image of a crosssectional lamella obtained by focused ion beam (FIB) milling at $y=400 \mu \mathrm{m}$. Thereby, we are able to compare $\mu$ GISAXS with a side view from one specific position of the gradient. The morphology obtained by TEM ( $\xi$ $=15.0 \pm 3.5 \mathrm{~nm}, H=7 \pm 1.6 \mathrm{~nm}, R=5.1 \pm 2.3 \mathrm{~nm})$ shows excellent agreement with that found in $\mu$ GISAXS ( $\xi$ $=18 \pm 2 \mathrm{~nm}, H=5.7 \pm 0.2 \mathrm{~nm}, R=5.7 \pm 2 \mathrm{~nm})$. Furthermore, TEM reveals the existence of interconnected Au nanoparticles on top of the PS layer, corroborated by a SEM image of the surface topography in plan view [fig. 3(b)]. This contrasts thick sputtered layers, showing vertical columnar structures. ${ }^{15}$ The interconnection of the nanoparticles breaks the confinement of the electron gas in the individual nanoparticles and thus the optical properties converge to the bulk properties. The characteristic plasmon resonance of nanoparticles with confined electron gas diminishes above the socalled critical thickness due to the interconnection. ${ }^{16}$ This can explain the similarity in the optical spectra in Fig. 1. Evaporated and sputtered Au layers of lower mass thickness do show differences in the optical spectra.

In region II (=lower sputter rate), with increasing distance $y$ from the beginning of the gradient, a transition from a coalescent nanoparticle layer to an isolated nanoparticle layer is observed. This leads to distinct changes in the $\mu$ GISAXS pattern as can be seen in Fig. 2(a) for $y_{1}$ $=620 \mu \mathrm{m}$ and $y_{2}=860 \mu \mathrm{m}$. In this region, we find a steep decrease of both height and radius with decreasing sputter rate. This demonstrates that during the initial stage of growth, coalescence does no longer take place and small particles prevail. As the distance between the nanoparticles decreases, the number of nanoparticles increases distinctly. The isolated nanoparticle layer now extends into region III. Here, only small but many, nm-sized clusters exist, coalescence is suppressed. The height continues to decrease, while radius and distance stay constant.

To conclude we performed a combinatorial investigation of a gradient sputtered metal nanoparticle layer using $\mu$ GISAXS in combination with local methods SEM and TEM. Although the optical properties of the sputtered sample are similar to the evaporated one with the same mass thickness, the nanoscale structure of both layers is distinctly different. In this case, the gradient is steeper as the sputter process has a lower divergence than evaporation. ${ }^{9}$ Furthermore, though the height of the nanoparticles is comparable, their in-plane structures are initially (region I) about 50\% larger in the case of sputtering. This can be due to the higher kinetic energy of sputtered $\mathrm{Au}$ atoms which enables them to diffuse larger distances on the PS surface. Furthermore, the shape of the nanoparticles differs distinctly for both coating techniques. In contrast to thick $\mu \mathrm{m}$-sized layers, the clusters are rather flat and do not grow into columnar structures. Hence, in the study presented here, we are looking at the initial stages of nanoparticle growth of sputtered gold. Specifically the transition of a coalescent layer to an isolated nanoparticle layer is obtained as a function of sputter rate.

The authors would like to thank I. Snigireva, A. Snigirev (ESRF Grenoble), J. Patommel (RWTH Aachen), and N. Hermsdorf (IPF Dresden) for their help during the experiment, and S. Pfister (TU München) for his help during the experiment and the data analysis.

${ }^{1}$ G. Mie, Ann. Phys. 25, 377 (1908).

${ }^{2}$ M. Valden, X. Lai, and D. W. Goodman, Science 281, 1647 (1998).

${ }^{3}$ M. Westphalen, U. Kreibig, J. Rostalski, H. Lüth, and D. Meissner, Sol. Energy Mater. Sol. Cells 61, 97 (2000).

${ }^{4}$ O. Stenzel, A. Stendal, K. Voigtsberger, and C. von Borczyskowski, Sol. Energy Mater. Sol. Cells 37, 337 (1995).

${ }^{5}$ M. Schmidt, R. Kusche, B. von Issendorff, and H. Haberland, Nature (London) 393, 238 (1998).

${ }^{6} \mathrm{G}$. Bauer, J. Hassmann, H. Walter, J. Haglmüller, C. Mayer, and T. Schalkhammer, Nanotechnology 14, 1289 (2003).

${ }^{7}$ T. L. Morkved, P. Wiltzius, H. M. Jaeger, D. G. Grier, and T. A. Witten, Appl. Phys. Lett. 64, 422 (1994).

${ }^{8}$ C. Schmitz, M. Thelakkat, and H.-W. Schmidt, Adv. Mater. (Weinheim, Ger.) 11, 821 (1999).

${ }^{9}$ S. V. Roth, M. Burghammer, C. Riekel, P. Müller-Buschbaum, A. Diethert, P. Panagiotou, and H. Walter, Appl. Phys. Lett. 82, 1935 (2003).

${ }^{10}$ C. Templier, S. Muzard, A. Galdikas, L. Pranevicius, J. Delafond, and J. C. Desoyer, Surf. Coat. Technol. 125, 129 (2000).

${ }^{11}$ F. Faupel, V. Zaporojtchenko, T. Strunskus, J. Erichsen, K. Dolgner, A. Thran, and M. Kiene, Metallization of Polymers 2, ACS Symposium Series (Kluwer Academic/Plenum, New York, 2002), p. 73.

${ }^{12}$ P. Müller-Buschbaum, S. V. Roth, M. Burghammer, A. Diethert, P. Panagiotou, and C. Riekel, Europhys. Lett. 61, 639 (2003).

${ }^{13}$ T. Salditt, T. H. Metzger, Ch. Brandt, U. Klemradt, and J. Peisl, Phys. Rev. B 51, 5617 (1995).

${ }^{14}$ R. Lazzari, J. Appl. Crystallogr. 35, 406 (2002).

${ }^{15}$ J. A. Thornton, Annu. Rev. Mater. Sci. 7, 239 (1977).

${ }^{16}$ C. Charton and M. Fahland, Surf. Coat. Technol. 142, 175 (2001). 